portal hypertension, and ascites even in dosages as low as 40000 units daily. Several hepatologists recommend high doses of vitamin A and in some cases zinc supplementation to prevent the ocular complications of primary biliary cirrhosis, suggesting up to 25000 units daily. ${ }^{4}$ Possibly those doses recommended might enhance hepatic damage in primary biliary cirrhosis.

By contrast with other studies, we could find no ocular abnormalities in any of our patients despite low circulating vitamin A concentrations, other studies finding as many as

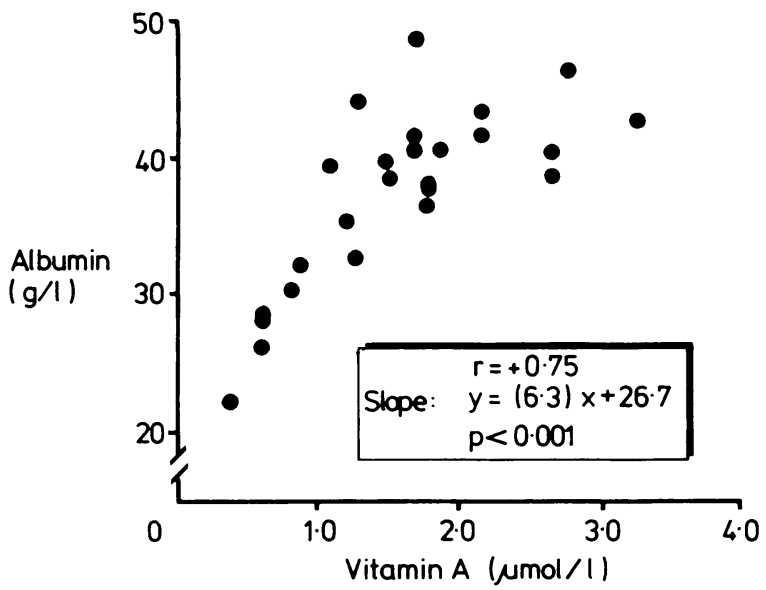

Correlation of vitamin A versus serum albumin concentrations Conversion: SI to traditional units-Vitamin A: $1 \mu \mathrm{mol} / 1 \approx$ $28 \cdot 7 \mu \mathrm{g} / 100 \mathrm{ml}$ nine out of 11 patients with primary biliary cirrhosis and clinical vitamin A deficiency. ${ }^{2}$ Vitamin $\mathrm{A}$ is transported to various tissues as a 1:1 molar complex with retinol binding proteins; as the concentration of these proteins falls so does the apparent value of vitamin A. In this study a serum albumin concentration of $<35 \mathrm{~g} / \mathrm{l}$ suggested the presence of a low circulating vitamin A concentration. Retinol binding protein and albumin production rely heavily on the synthetic capacity of the liver, which is disturbed in primary biliary cirrhosis. Hence despite low recordable vitamin A values the tissue concentrations may be normal. In addition, the rare use of cholestyramine and a normal dietary fat intake in our patients allowed a normal dietary vitamin A intake.

Our data suggest that regular vitamin A supplementation is unnecessary for all patients with primary biliary cirrhosis. A serum albumin concentration of $<35 \mathrm{~g} / 1$ is a level below which yearly ocular evaluation should be considered to assess the need for vitamin A supplementation.

\section{References}

1 Russell R, Morrison S, Smith F, Oaks E, Carney E. Vitamin A reversal of abnormal dark adaptation in cirrhosis. Ann Intern Med 1978;88:622-6.

Herlong $\mathrm{H}$, Russell R, Maddrey W. Vitamin A and zinc therapy in primary biliary cirrhosis. Hepatology $1981 ; 1: 348-51$

3 Sherlock S. Primary biliary cirrhosis: critical evaluation and treatment policies. Scand $\mathcal{F}$ Gastroenterol 1982 ;supp 17 7:63-74

( night blindness in primary biliary cirrhosis. Br Med f 1984;288:1030-1.

5 Stewart WK, Fleming LW. Plasma retinol and retinol binding protein concentration in patients on maintenance haemodialysis with and without vitamin supplements. Nephron 1982;30:15-21.

(Accepted 12 September 1984)

\title{
Factors affecting development of peritonitis in continuous ambulatory peritoneal dialysis
}

\author{
T H J GOODSHIP, A HEATON, R S C RODGER, M K WARD, R WILKINSON， D N S KERR
}

\begin{abstract}
A questionnaire based survey in patients receiving continuous ambulatory peritoneal dialysis showed that there was an increased incidence of upper respiratory tract symptoms (suggestive of viral illness) in the 14 days before the development of peritonitis. No other factors were identified that might distinguish patients who develop peritonitis.

The possibility that viral infections predispose to peritonitis by altering host defence mechanisms in patients receiving this form of renal replacement therapy warrants further study.
\end{abstract}

\section{Introduction}

Continuous ambulatory peritoneal dialysis is now a well established method of renal replacement therapy. Since its introduction in $1978^{1}$ the major complication has remained that of peritonitis. Although in most renal units using the treatment there has been a fall in the incidence of peritonitis, it is still the main source of morbidity. ${ }^{2}$ Most infections are caused by skin commensals, and it is suspected that most episodes are secondary to a breakdown in technique.

In an attempt to elucidate some of the factors that may be important in the development of peritonitis we have carried out a survey on the methods of the exchange technique used by our patients and their association with the development of peritonitis.

\section{Patients and methods}

At the beginning of December 1982 all 97 patients who were being treated by continuous ambulatory peritoneal dialysis and were free of peritonitis were sent a questionnaire. Replies from 94 patients were received. All patients who subsequently joined the treatment programme over the next five months were also given the questionnaire. The questionnaire consisted of two parts (see Appendix). Questions in the first part asked about general hygiene and methods used in the exchange technique, and the second part asked about problems that had occurred in the previous two weeks. 
All patients who subsequently developed peritonitis were given the second part of the questionnaire again when they presented with peritonitis. They were allowed to take the questionnaire home, returning it later by post, and it was not compulsory for them to disclose their identity. We hoped that this would increase the veracity of their answers, especially with regard to contamination. In this way we were able to compare a normal two week period of continuous ambulatory peritoneal dialysis with a two week period before the development of peritonitis. During the five months December 1982 to the end of April 1983 there were 47 episodes of peritonitis in 37 patients, 10 patients having two episodes. This represented a rate of one episode per 10.3 patient months. In 12 of the episodes the patient did not identify himself or herself on the questionnaire; although we knew which 12 patients these were, we could not match questionnaire to individual patient.

Statistical analysis-Patients who never developed peritonitis were compared by $\chi^{2}$ test with those who at some stage in the course of the study did develop the complication. In order to compare the incidence of upper respiratory tract symptoms in the two weeks before the development of peritonitis with another two week period in the same patient we used McNemar's test for paired proportions, with a continuity correction. ${ }^{3}$ In patients who had more than one episode of peritonitis only the first episode was used for this analysis. It was thus possible to analyse 28 matched two week periods in the same patients.

\section{Results}

Analysis of the replies to the first part of the questionnaire (Appendix) showed no significant patterns distinguishing patients who later developed peritonitis from those who did not.

In analysing the second part of the questionnaire we looked for those factors which might predict the development of peritonitis. There was an increase in the incidence of upper respiratory tract symptoms from $31.7 \%$ in a normai two week period to $51.2 \%$ in the two weeks before the development of peritonitis (table I); this increase just failed to reach significance at the $5 \%$ level $(p=0.07)$. There was no difference in the incidence of upper respiratory tract symptoms in two week periods, not followed by peritonitis, between patients who later developed peritonitis and those who did not (table II).

TABLE I-Incidence (number of episodes) of upper respiratory tract symptoms in 28 matched two week periods in same patients (data arranged as contingency table)

\begin{tabular}{llcc}
\hline & \multicolumn{2}{c}{ Before peritonitis } \\
\cline { 3 - 3 } Not before peritonitis & No cold & Cold \\
\cline { 2 - 3 } & $\left\{\begin{array}{lcc}\text { No cold } \\
\text { Cold }\end{array}\right.$ & 10 & 9 \\
& 2 & 7
\end{tabular}

$u=1 \cdot 81 ; p=0.07$.

TABLE II-Incidence of upper respiratory tract symptoms (number of patients) in normal two week period (data arranged as contingency table)

\begin{tabular}{lcc}
\hline & Cold & No cold \\
\hline Patients who never developed peritonitis & 18 & $39\left(31.5^{\circ}\right)$ \\
Patients who developed peritonitis during study & 10 & $24\left(29 \cdot 4^{\circ}\right)$ \\
\hline
\end{tabular}

$x^{2}=0.0470 ; 0.90>p>0.80$

\section{Discussion}

There are several possible explanations for the increased incidence of upper respiratory tract symptoms before the development of peritonitis. The patients may have been misinterpreting the prodromes of peritonitis as those of a "cold." There may be an increased risk of contamination owing to lack of concentration during the general malaise associated with an upper respiratory tract infection. Viral illness may alter the host defence mechanisms responsible for overcoming bacterial infections. If this is the explanation it implies that a small number of bacteria regularly reach the peritoneal cavity but are eliminated by the natural defence mechanisms. In any analysis of multiple factors increased significance associated with one might by itself be a chance finding. This possibility can be ruled out only by replication of the study.

Whether our finding calls for any intervention will not be clear until a prospective controlled trial has been carried out. Nevertheless, we do know of one continuous ambulatory peritoneal dialysis unit in which patients with upper respiratory tract infections are given prophylactic intraperitoneal antibiotics.

Our study has failed to detect any characteristics of the patient, any variations in technique, or other factors that might predict the likelihood of development of peritonitis, other than the occurrence of symptoms suggesting upper respiratory infection during the preceding two weeks. If faulty technique is the major factor responsible for the development of peritonitis it usually goes unrecognised by the patient. The possibility that viral infections predispose to peritonitis by altering host defence mechanisms merits further study.

\section{Appendix}

TOPICS COVERED IN FIRST AND SECOND PARTS OF QUESTIONNAIRE

First part

Sex

Age

System used for continuous ambulatory peritoneal dialysis (all patients using Travenol)

Method of washing hands before exchanges

Surface on which exchanges were performed

Cleaning of exchange surface

Performance of exchanges away from home

Checks undertaken before exchange-for example, no leaks in bags, new bag not cloudy

Method of draining out and in

Frequency of dressings

Use of gloves for exchanges

Storage of bag after draining in

Whether anybody else did patient's exchanges

Second part

Contamination of spike or screw

Contamination of gloves

Infection of exit site

Disconnection of set

Leakage either from set or from Tenckhoff catheter

Delay in either running in or running out

Occurrence of diarrhoea, constipation, flu, cold, or skin infection

\section{References}

1 Oreopoulos DG, Robson M, Izatt S, et al. A simple and safe technique for continuous ambulatory peritoneal dialysis (CAPD). Trans Am Soc Artif Intern tinuous ambulatory pert

2 Gokal R, Ramos JM, Francis DMA, et al. Peritonitis in continuous ambulatory 3 Armitage P. Statistical methods in medical research. London: Blackwell, 1971:127.

(Accepted 2 October 1984)

\section{ONE HUNDRED YEARS AGO}

A telegram from Dongola, through Reuter's agency, under date of November 8th, says:-Lord Wolseley to-day inspected the fieldhospital, and expressed himself much pleased with the arrangements for the comfort of the invalids made by Surgeon-Major Ferguson and the Medical Staff. He especially praised the spacious cool straw huts in which the sick are accommodated. There are at present fifty-six cases of sickness under treatment, but none of them are of a serious character. (British Medical fournal 1884 ;ii:972.) 